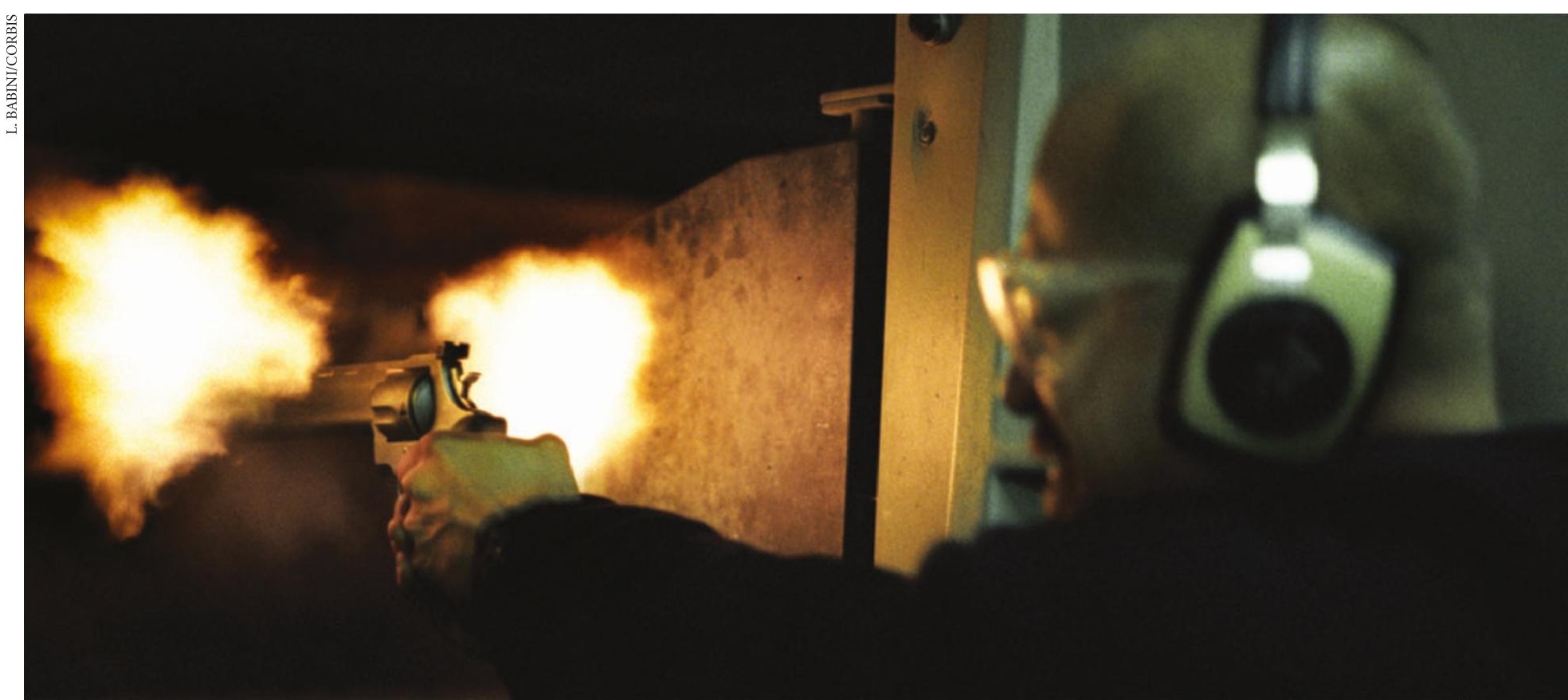

Eat lead: toxins from explosives can reach dangerously high levels at the firing ranges where soldiers and police are trained.

\title{
Collateral damage
}

\section{Even munitions that are never used in anger can have a long-term impact on the environment, and the military is anxious to minimize the risks. Jim Giles talks to the chemists who are developing 'green' explosives.}

" know ... some people think it is an oxymoron." Ron Jones sounds weary. He has had to deal with my incredulous line of questioning before. "But we really do need green explosives."

Bombing and shelling can't be good for any ecosystem. But away from the heat of battle, military officials are ploughing precious research funds into developing lesstoxic explosives that should be better both for the personnel who have to handle them, and for the environment.

Jones and his colleagues at the US Naval Air Warfare Center in China Lake, California, for instance, are trying to replace the lead-based compounds used to fire guns. Other groups want to phase out explosives whose residues not only pollute the environment when detonated, but also cause problems when unused ordnance is disposed of. "The environmental issues with weapons go beyond what you see in combat," says Jones.

Pollution is a particularly pressing issue at the firing ranges where soldiers and lawenforcement officers are trained.

When you pull the trigger of a firearm, two small explosions follow. First a hammer or an electrical current detonates a small amount of 'primer' explosive. This ignites a larger amount of explosive, which forces the bullet down the barrel.
The problem is that the most widely used primers contain lead. Two of the current favourites - lead azide and lead styphnate - are responsible for the dangerously high levels of lead found at some firing ranges. A 1991 survey, for instance, found that employees who had just cleaned a range run by the FBI in Quantico, Virginia, had levels of lead in their blood almost ten times as high as US government health limits ${ }^{1}$.

\section{Target practice}

Jones and his colleagues hope to replace lead compounds with nanoparticles of aluminium, which react violently with oxygen. They mix particles averaging 50 nanometres across with acetylene black, a form of carbon, and molybdenum trioxide. The former makes the mixture electrically conductive, allowing it to be ignited by passing a current through it. The latter supplies oxygen.

The researchers are now running tests to assess 'action times' - the gap between the ignition current switching on and the bullet leaving the muzzle of the gun, which for military purposes needs to be less than 4 milliseconds. If the aluminium particles pass this and other assessments, they could be in use sometime in the next decade.

At Ludwig-Maximilians University in Munich, Germany, chemists led by Thomas
Klapötke have investigated about 50 substances that could make safer primers, funded largely by the German military. A primer must detonate relatively easily to ensure that guns can fire quickly, so the researchers first measure how much energy it takes to detonate them. They do this by rubbing samples between sandpaper, dropping weights on them and spraying them with sparks.

Next up are tests that measure destructive power. About 10 grams of the test substance is placed in a standard steel container, then ignited. Each container has a hole of a particular size in the lid. The smaller the hole, the more likely it is that gases produced by the explosion will be unable to escape quickly enough, blowing the container apart. The ideal primer - which only needs to be powerful enough to ignite the secondary explosive - will destroy a 35-millilitre container with a hole in the lid 4 millimetres across.

In five years of testing only five compounds, all of them rich in nitrogen, have made it through this process. One candidate, known as TNTA, consists of a series of $\mathrm{N}_{3}$ and $\mathrm{NO}_{2}$ groups attached to a ring of carbon atoms ${ }^{2}$. Like other nitrogen-based explosives, part of its energy release comes from single or double bonds between nitrogen atoms that break apart and re-form as more stable triple bonds. When mixed with an 
for defence laboratories in the US Department of Defense, says that the US military has millions of kilograms of explosives that have reached the end of their shelf-life of about 20-50 years, but which are difficult to dispose of because of environmental concerns.

The explosives involved - nitrogen and oxygen-containing substances called HMX and RDX - are mixed with polymers so that they can easily be moulded into various shapes for different applications. The polymer-explosive mix could be disposed of by burning, which does not necessarily raise it to a temperature at which it would explode.

Short argues that the environmental risks are not large. But communities living close to military facilities have blocked such moves, fearing that burning the polymers could release pollutants such as carbon monoxide. Military officials have an added incentive to replace HMX and RDX: they want explosives that are cheap and powerful, yet detonate less readily and so are easier to handle.

In the 1990s, Short was part of a team at the Office of Naval Research in Arlington, Virginia, that investigated alternatives to conventional explosive-polymer mixes. The group found that HMX and RDX could be mixed with polymers that melt when heated, allowing the explosives to be removed and stored for future use. Short's team also studied a new explosive, a nitrogenrich compound called TNAZ, which can itself be melted and moulded ${ }^{3}$.

But money was an but the environmental effects take a little longer to kick in. utes of a space shuttle launch, for instance, when the need for thrust is greatest, power is generated by burning aluminium in two solid-fuel boosters. The aluminium is supplied with oxygen using a compound called ammonium perchlorate, but environmentalists note that this means the exhaust plume contains chlorine ions - which can trigger ozone-destroying reactions and acid rain.

Space agencies are continually searching for more efficient propellants and, although they believe that the environmental impact of the chlorine ions is minimal, they take such considerations into account. Three main candidates - known as ADN, HNF and CL-20 - are in the frame to replace ammonium perchlorate as an oxidizer. All are chlorine-free, consisting mainly of nitrogen and oxygen.

\section{Blast off:}

Results from tests in military and civilian labs in Europe and the United States suggest that they could improve the performance of solid-fuel boosters. But introducing a new propellant is a complex business, says Octavia Frota, an expert in energetic materials at ESTEC, the European Space Agency's research and technology-development centre in Noordwijk, the Netherlands. Tests to find the right combination of fuel and oxidizer, and a compound to bind them together, are subject to stringent safety regulations. Boosters may have to be redesigned once the right combination is found, she adds.

It could take another decade for any of the three candidate oxidizers to find regular space applications. But thanks to the presence of nitrogen in these compounds, they can act as explosives as well as oxidizers. And at least one - CL-20 - is being assessed for use as an explosive by military researchers in France, Germany and Britain, says Klapötke.

Working to develop new explosives can be dangerous. "If you make one mistake it can be your last," says Klapötke. Three years ago, one researcher in his lab lost the top halves of two fingers in an explosion. "But he is still working in the field," says Klapötke.

Labs such as his can ill afford to lose staff, due to problems with recruitment. Klapötke admits that military links are off-putting for many students. And the pool of recruits is narrowed still further by his decision only to accept students from NATO countries for security reasons. Klapötke understands why some young chemists shy away from military research, but argues that his work is necessary. "We need defence, so we have to train people," he says. "But we don't want to kill them and pollute the environment."

\section{$\mathrm{Jim}$ Giles is a reporter for Nature, based in London.}

1. Barsan, M. E. \& Miller, A. Lead Health Hazard Evaluation HETA Report No. 91-0346-2572 (National Institute for Occupational Safety and Health, Cincinnati, Ohio, 1996)

2. Adam, D., Karaghiosoff, K. \& Klapötke, T. M. Propell. Explos. Pyrot. 27, 7-11 (2001).

3. Archibald, T. G., Gilardi, R., Baum, K. \& George, C.

J. Org. Chem. 55, 2920-2924 (1990).
James Short, a staff specialist
Jare can be dangerous. Three years ago, a researcher lost the top halves of two fingers in an explosion - but he's still working in the field." greener alternatives to the propellants used to power military rockets and space vehicles.

During the first two min-

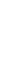

